\title{
CRYSTAL STRUCTURE AND HIRSHFELD SURFACE ANALYSIS OF (E)-5-PHENYL-3-((4- (TRIFLUOROMETHYL)BENZYLIDENE)AMINO)THIAZOLIDIN-2-IMINIUM BROMIDE
}

\author{
ABEL M. MAHARRAMOV ${ }^{A}$ GULNARA SH. DURUSKARII, ${ }^{A}$ GUNAY Z. MAMMADOVA ${ }^{A}$ ALI N. \\ KHALILOV,${ }^{A, B}$ JAVAHIR M. ASLANOVA, ${ }^{A}$ JONATHAN CISTERNA, ${ }^{C}$ ALEJANDRO CÁRDENAS, ${ }^{D}$ IVÁN BRITO ${ }^{*}$ \\ ${ }^{a}$ Organic Chemistry Department, Baku State University, Z. Xalilov Str. 23, AZ 1148, Baku, Azerbaijan. \\ ${ }^{b}$ Department of Physics and Chemistry, "Composite Materials" Scientific Research Center, Azerbaijan State Economic University \\ (UNEC), H.Aliyev str. 135, Az 1063, Baku, Azerbaijan \\ ${ }^{c}$ Departamento de Química, Facultad de Ciencias Básicas, Universidad de Antofagasta, Avda. Universidad de Antofagasta 02800 \\ Campus Coloso, Antofagasta, Chile. \\ ${ }^{d}$ Departamento de Física, Facultad de Ciencias Básicas, Universidad de Antofagasta, Avda. Universidad de Antofagasta 02800, \\ Campus Coloso, Antofagasta, Chile.
}

\section{ABSTRACT}

In the cation of the title salt, the central thiazolidine ring adopts an envelope conformation. In the crystal $\mathrm{N}-\mathrm{H} \cdots \mathrm{Br}$ hydrogen bonds link the components into a bi-dimensional network with the cations and anions stacked parallel to plane (101). The molecular structure shows several positional disorders over $-\mathrm{CF}_{3}$ and thiazolidine fragments and these were modeled. The weak intermolecular interactions in the crystal structure are mainly constituted by $\mathrm{H} \cdots \mathrm{F}, \mathrm{H} \cdots \pi$ and $\mathrm{H} \cdots \mathrm{Br}$. Hirshfeld surface analysis were used to verify the contributions of the different intermolecular interactions.

\section{INTRODUCTION}

Schiff bases and related N-ligands attract considerable interest and play an important role in the development of the chemistry of chelate systems as potential ligands towards a large number of metal ions ${ }^{1-8}$. Growing interest in the synthesis of such a type of compounds are due to their potential application as catalyst in many organic transformations ${ }^{9-12}$ and diverse useful properties, such as solvatochromism $^{13}$, molecular switching ${ }^{14,15}$, crystal engineering ${ }^{16}$, etc. Noncovalent interactions (hydrogen, aerogen, halogen, chalcogen, pnictogen, tetrel and icosagen bonds, as well as cation- $\pi$, anion- $\pi$, lone pair- $\pi, \pi-\pi$ stacking, agostic, pseudoagostic, anagostic, dispersion-driven, lipophilic, etc.) concern weak forces of attraction formed between different molecules (intermolecular) or fragments of the same molecule (intramolecular). While these weak interactions were firstly taken into consideration by van der Waals in $1873^{17}$, the understanding of their crucial role in synthesis, catalysis, crystal engineering, pharmaceutical design, molecular biology, molecular recognition, materials, etc. has been increasingly explored in the last few decades.

Herein we found strong charge assisted hydrogen bond and halogen bonding in (E)-5-phenyl-3-((4-(trifluoromethyl)benzylidene)amino)thiazolidin-2iminium bromide (scheme 1).<smiles></smiles>

Scheme 1.

\section{EXPERIMENTAL}

NMR spectra were recorded at room temperature on a Bruker Avance II + 300 (UltraShield $^{\mathrm{TM}}$ Magnet) spectrometer operating at 300.130 and $75.468 \mathrm{MHz}$ for proton and carbon-13, respectively. All NMR spectra are reported in parts per million (ppm, d) relative to tetramethylsilane $\left(\mathrm{Me}_{4} \mathrm{Si}\right.$ ) for ${ }^{1} \mathrm{H}$ and ${ }^{13} \mathrm{C} \mathrm{NMR}$ spectra, with the residual solvent proton and carbon resonances used as internal standards. Coupling constants $(\mathrm{J})$ are reported in Hertz $(\mathrm{Hz})$, and integrations are reported as number of protons.

The following abbreviations are used to describe peak patterns: $\mathrm{s}=$ singlet, $\mathrm{d}=$ doublet, $\mathrm{t}=$ triplet, $\mathrm{m}=$ multiplet, $\mathrm{br}=$ broad. ${ }^{1} \mathrm{H}$ and ${ }^{13} \mathrm{C}$ NMR chemical shift assignments are supported by data obtained from ${ }^{1} \mathrm{H}-{ }^{1} \mathrm{H}$ COSY,${ }^{1} \mathrm{H}-{ }^{13} \mathrm{C} \mathrm{HMQC}$, and ${ }^{1} \mathrm{H}-{ }^{13} \mathrm{C}$. Electrospray mass spectra (ESI-MS) were run with an ion-trap instrument (Varian 500-MS LC Ion Trap Mass Spectrometer) equipped with an electrospray ion source. For electrospray ionization, the drying gas and flow rate were optimized according to the particular sample with 35 p.s.i. nebulizer pressure. Scanning was performed from $m / z 0$ to 1100 in methanol solution. The compounds were observed in the positive mode (capillary voltage $=80-105 \mathrm{~V}$ ).

For the molecular structure of title compound, $\mathrm{H}$ atoms were located in the difference Fourier map, refined with fixed individual displacement parameters, using a riding model with $\mathrm{C}-\mathrm{H}$ distances of $0.93 \AA$ (for aromatic rings), $0.92 \AA$; $0.96 \AA$ (for $\mathrm{CH}_{3}, \mathrm{CH}_{2}$ ), with $\mathrm{U}(\mathrm{H})$ values of $1.2 \mathrm{U}_{\mathrm{eq}}(\mathrm{C}, \mathrm{N})$ (for $\mathrm{CH}$ in aromatic rings and $\left.-\mathrm{NH}_{2}^{+}\right)$, and $1.5 \mathrm{U}_{\mathrm{eq}}(\mathrm{C}, \mathrm{O})\left(\right.$ for $\mathrm{CH}_{3}$ and $\left.-\mathrm{OH}\right)$. Solvent molecules were restrained using Rigid body (RIGU) restrains (O1S, C1S). trifluoromethyl moiety $\left(-\mathrm{CF}_{3}\right)$ was restrained using SADI. All sigma for 1-2 distances of 0.004 $\AA$ and sigma for $1-3$ distances of $0.004 \AA$. Finally the several disordered $-\mathrm{CF}_{3}$, was treated with FVAR and SUMP.

X-ray diffraction patterns of title compound were collected using a Bruker SMART APEX-II CCD area detector equipped with graphite-monochromated Mo-K $\alpha$ radiation $(\lambda=0.71073 \AA)$ at room temperature. The diffraction frames were integrated using the APEX3 package ${ }^{18}$. The structure of were solved by intrinsic phasing ${ }^{19}$ using the OLEX 2 program ${ }^{20}$.

The structure was then refined with full-matrix least-square methods based on $F^{2}$ (SHELXL-2014) ${ }^{19}$. For $\mathrm{C}_{14} \mathrm{H}_{8} \mathrm{BrCl}_{2} \mathrm{FN}_{2}$, non-hydrogen atoms were refined with anisotropic displacement parameters. All hydrogen atoms were included in their calculated positions, assigned fixed isotropic thermal parameters and constrained to ride on their parent atoms. A summary of the details about crystal data, collection parameters and refinement are documented in Table 1, and additional crystallographic details are in the CIF files. ORTEP views were drawn using OLEX2 software $^{20}$. CCDC 1912059 contain the supplementary crystallographic data for this paper. These data can be obtained free of charge from the Cambridge Crystallographic Data Centre via www.ccdc.cam.as.uk/data request/cif. 
Table 1. Crystal data parameters for title compound.

\begin{tabular}{|c|c|}
\hline Empirical Formula & $\mathrm{C}_{18} \mathrm{H}_{21} \mathrm{BrF}_{3} \mathrm{~N}_{3} \mathrm{O}_{2} \mathrm{~S}$ \\
\hline Formula mass, $\mathrm{g} \mathrm{mol}^{-1}$ & 480.35 \\
\hline Collection T, K & 296.15 \\
\hline crystal system & monoclinic \\
\hline space group & $\mathrm{P} 2{ }_{1} / \mathrm{n}$ \\
\hline$a(\AA)$ & $5.7935(3)$ \\
\hline$b(\AA)$ & $11.6768(9)$ \\
\hline$c(\AA)$ & $31.310(2)$ \\
\hline$\beta\left(^{\circ}\right)$ & $95.259(4)$ \\
\hline$V\left(\AA^{3}\right)$ & $2109.2(2)$ \\
\hline$Z$ & 4 \\
\hline$\rho_{\text {calcd }}\left(\mathrm{gcm}^{-3}\right)$ & 1.513 \\
\hline Crystal size $(\mathrm{mm})$ & $0.52 \times 0.31 \times 0.17$ \\
\hline$F(000)$ & 976.0 \\
\hline $\operatorname{abs} \operatorname{coeff}\left(\mathrm{mm}^{-1}\right)$ & 2.092 \\
\hline $2 \theta$ range $\left({ }^{\circ}\right)$ & $2.612-56.186$ \\
\hline range h,k,l & $-7 / 7,-14 / 15,-41 / 41$ \\
\hline No. total refl. & 16972 \\
\hline No. unique refl. & $5122\left[\mathrm{R}_{\text {int }}=0.0504, \mathrm{R}_{\text {sigma }}=0.0613\right]$ \\
\hline Comp. $\theta_{\max }(\%)$ & $99.5 / 28.09$ \\
\hline Data/Restraints/Parameters & $5122 / 41 / 279$ \\
\hline Final R $[I>2 \sigma(I)]$ & $\mathrm{R}_{1}=0.0783, \mathrm{wR}_{2}=0.1938$ \\
\hline $\mathrm{R}$ indices (all data) & $\mathrm{R}_{1}=0.1057, \mathrm{wR}_{2}=0.2136$ \\
\hline Goodness of fit / $\mathrm{F}^{2}$ & 1.132 \\
\hline Largest diff. Peak/hole $\left(\mathrm{e}^{-3}\right)$ & $1.52 /-0.86$ \\
\hline
\end{tabular}

To the solution of $1 \mathrm{mmol}$ of 3-amino-5-phenylthiazolidin-2-iminium bromide in $20 \mathrm{~mL}$ ethanol was added $1 \mathrm{mmol}$ of 4-(trifluoromethyl)benzaldehyde and refluxed for 2 hours. Then the reaction mixture was cooled down. Reaction products was precipitated from reaction mixture as a colorless single crystals, collected by filtration and washed with cold acetone.Yield $73 \%$. Mp $231^{\circ} \mathrm{C}$. Anal. Calcd. for $\mathrm{C}_{17} \mathrm{H}_{15} \mathrm{BrF}_{3} \mathrm{~N}_{3} \mathrm{~S}(M r=430.29)$ : C, 47.45; H, 3.51; N, 9.77. Found: $\mathrm{C}$, 47.40; H, 3.48; N, $9.71 \% .{ }^{1} \mathrm{H}$ NMR $\left(300 \mathrm{MHz}, \mathrm{DMSO}-d_{6}\right): 4.58\left(\mathrm{k}, 1 \mathrm{H}, \mathrm{CH}_{2}{ }^{3} \mathrm{~J}_{\mathrm{H}}\right.$ $\left.{ }_{\mathrm{H}}=6.6\right) ; 4,89\left(\mathrm{t}, 1 \mathrm{H}, \mathrm{CH}_{2},{ }^{3} \mathrm{~J}_{\mathrm{H}-\mathrm{H}}=8.4\right) ; 5.60\left(\mathrm{t}, 1 \mathrm{H}, \mathrm{CH}-\mathrm{Ar},{ }^{3} \mathrm{~J}_{\mathrm{H}-\mathrm{H}}=7.5\right) ; 7.39-8.29$ $(\mathrm{m}, 9 \mathrm{H}, 9 \mathrm{Ar}-\mathrm{H}) ; 8.51(\mathrm{~s}, 1 \mathrm{H}, \mathrm{CH}=) ; 10.51(\mathrm{~s}, 1 \mathrm{H}, \mathrm{NH}=) .{ }^{13} \mathrm{C} \mathrm{NMR}(75 \mathrm{MHz}$, DMSO- $\left.d_{6}\right): 45.45,56.03,125.74,125.80,127.86,128.95,129.15,129.22$,
$130.72,131.14,136.85,137.50,149.54,168.62$. MS (ESI), $\mathrm{m} / \mathrm{z}: 350.38$ $\left[\mathrm{C}_{17} \mathrm{H}_{15} \mathrm{~F}_{3} \mathrm{~N}_{3} \mathrm{~S}\right]^{+}$and $79.88 \mathrm{Br}^{-}$.

\section{RESULTS AND DISCUSSION.}

The cation structure corresponds to the $E$ isomer in the solid state. All the distances and angles are normal ${ }^{21,22}$. The bond lengths range between measured $\mathrm{C}-\mathrm{C}$ and $\mathrm{C}=\mathrm{N}$ values for single and double bonds in $\mathrm{C} 4-\mathrm{C} 5, \mathrm{C} 5-\mathrm{N} 1$ and $\mathrm{N} 1-\mathrm{N} 2$ are slightly larger than the reported for similar organic compounds ${ }^{23-25}$, depicted a potential electronic disconnection between the rings in the cation of the title compound.

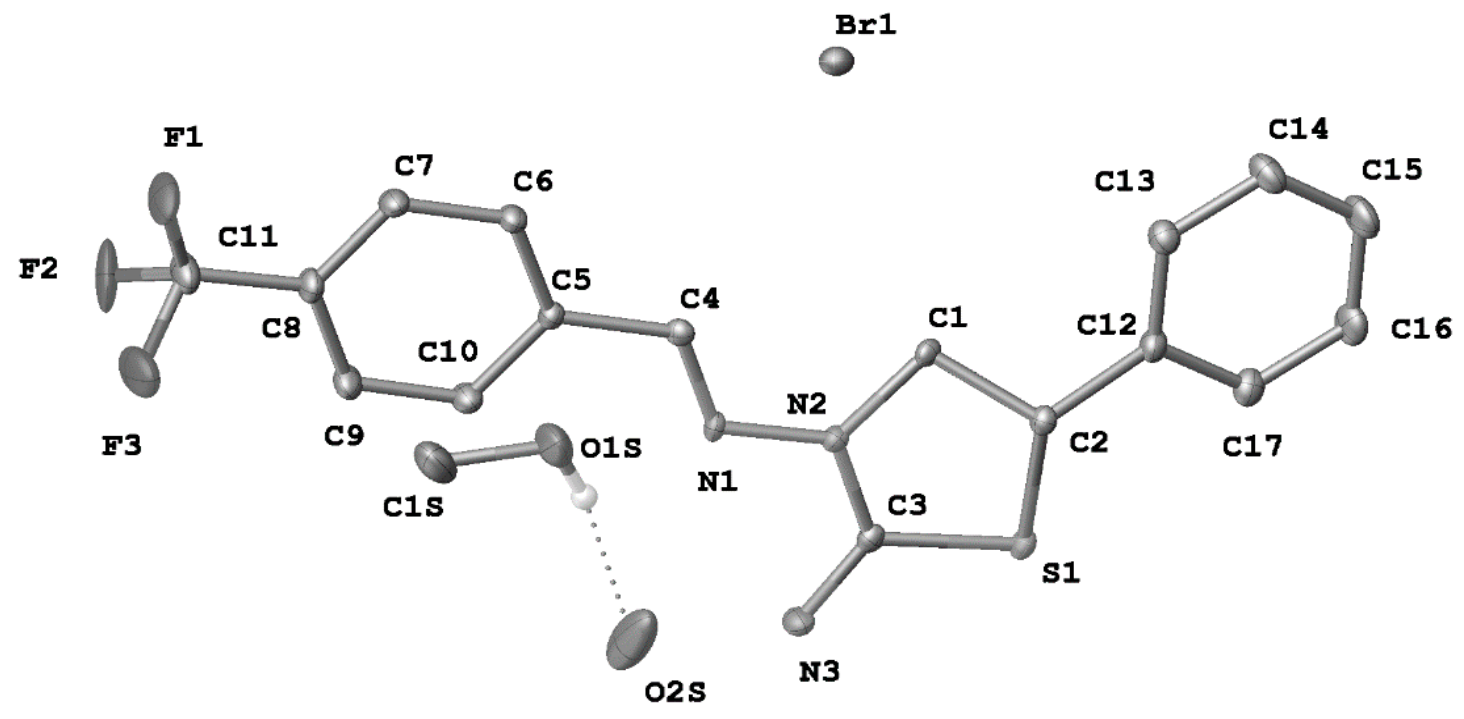

Figure 1. ORTEP plot of the title compound. Thermal ellipsoid was drawn with $30 \%$ of probability. Some hydrogen atoms were omitted for sake.

In the cation of the title salt, the central thiazolidine ring $(\mathrm{S} 1 / \mathrm{N} 2 / \mathrm{C} 1-\mathrm{C} 3)$ adopts an envelope conformation with puckering parameters $0.300(6) \AA$, and $\varphi(2)=$ $325,1(12)^{\circ}$. The dihedral angle between the mean plane of the central thiazolidine ring and the (4-trifluoromethyl)benzylidene ring (C5-C10) is $0.4(3)$ while this plane make a angle $10(2)^{\circ}$ with the phenyl ring. The N2-N1-C4-C5 bridge that links the thiazolidine and (4-trifluoromethyl)benzylidene ring is $0.40(3)^{\circ}$ (see tables 3 and 4). 
J. Chil. Chem. Soc., 64, N² (2019)

Table 2. Fractional Atomic Coordinates $\left(\times 10^{4}\right)$ and Equivalent Isotropic Displacement Parameters $\left(\AA^{2} \times 10^{3}\right)$ for title compound. $U_{\text {eq }}$ is defined as $1 / 3$ of of the trace of the orthogonalised $\mathrm{U}_{\mathrm{IJ}}$ tensor.

\begin{tabular}{|c|c|c|c|c|}
\hline Atoms & $x$ & $y$ & $z$ & $\mathrm{U}(\mathrm{eq})$ \\
\hline Br1 & $14384.5(10)$ & $2642.6(5)$ & $7630.0(2)$ & $31.7(2)$ \\
\hline $\mathrm{S} 1$ & $5698(2)$ & $5952.5(13)$ & 7912.1(4) & $28.4(3)$ \\
\hline F1A & 13725(9) & 2291(6) & $5137.4(16)$ & $72(2)$ \\
\hline F2A & $9940(40)$ & $1910(20)$ & $4957(6)$ & $92(6)$ \\
\hline F3A & $11190(20)$ & $3418(9)$ & $4903(2)$ & $97(4)$ \\
\hline F1B & $13110(80)$ & $3170(50)$ & $5005(14)$ & $72(2)$ \\
\hline F2B & $9970(50)$ & $2870(30)$ & $4861(8)$ & $97(4)$ \\
\hline F3B & $10770(40)$ & $1435(13)$ & $5094(5)$ & $92(6)$ \\
\hline O1S & $4010(20)$ & $6087(10)$ & $6154(2)$ & $158(6)$ \\
\hline N1 & $8072(8)$ & $4562(4)$ & $6923.8(13)$ & $24.0(10)$ \\
\hline $\mathrm{N} 2$ & $7800(8)$ & $4922(4)$ & $7335.3(15)$ & $24.8(10)$ \\
\hline N3 & $4486(9)$ & $5894(5)$ & $7069.8(16)$ & $35.7(12)$ \\
\hline $\mathrm{C} 1$ & $9231(9)$ & $4612(5)$ & $7724.9(17)$ & $24.5(11)$ \\
\hline $\mathrm{C} 3$ & $5958(9)$ & $5579(5)$ & $7384.4(17)$ & $25.6(11)$ \\
\hline $\mathrm{C} 4$ & $9802(10)$ & $3931(5)$ & $6872.6(18)$ & $26.0(11)$ \\
\hline $\mathrm{C} 5$ & 10177(9) & $3531(5)$ & $6437.9(16)$ & $25.2(11)$ \\
\hline C6 & $12186(10)$ & $2943(5)$ & $6368.8(18)$ & $31.9(13)$ \\
\hline $\mathrm{C} 7$ & $12622(11)$ & $2597(6)$ & 5964.3(19) & $35.2(14)$ \\
\hline $\mathrm{C} 8$ & $11035(11)$ & $2845(6)$ & $5616.9(18)$ & $38.1(15)$ \\
\hline C9 & $8995(11)$ & $3414(6)$ & 5682.1(19) & $40.3(16)$ \\
\hline $\mathrm{C} 10$ & $8574(11)$ & $3770(6)$ & $6086.6(19)$ & $37.7(15)$ \\
\hline $\mathrm{C} 11$ & $11509(12)$ & $2527(7)$ & $5183(2)$ & $55(2)$ \\
\hline $\mathrm{C} 12$ & $9042(10)$ & $5043(6)$ & $8521.8(18)$ & $35.1(14)$ \\
\hline $\mathrm{C} 13$ & $11095(10)$ & $4464(6)$ & $8637.2(18)$ & $35.9(14)$ \\
\hline $\mathrm{C} 14$ & $11758(12)$ & $4214(7)$ & $9060(2)$ & $49.8(19)$ \\
\hline $\mathrm{C} 15$ & $10333(14)$ & $4513(8)$ & $9372(2)$ & $59(2)$ \\
\hline $\mathrm{C} 16$ & $8294(13)$ & $5090(7)$ & $9259(2)$ & 49.4(19) \\
\hline $\mathrm{C} 17$ & $7633(11)$ & $5337(6)$ & 8841.2(19) & $39.4(15)$ \\
\hline $\mathrm{C} 2 \mathrm{~A}$ & $8686(13)$ & $5497(7)$ & $8052(2)$ & $25.9(15)$ \\
\hline $\mathrm{C} 2 \mathrm{~B}$ & $7620(30)$ & $4718(18)$ & $8105(6)$ & $25.9(15)$ \\
\hline C1S & $3780(20)$ & $5914(9)$ & $5728(3)$ & $88(4)$ \\
\hline $\mathrm{O} 2 \mathrm{~S}$ & $9430(30)$ & $7111(8)$ & $6313(3)$ & $169(6)$ \\
\hline
\end{tabular}


Table 3. Bond Angles for the title compound.

\begin{tabular}{|c|c|c|c|c|c|c|c|}
\hline \multicolumn{3}{|c|}{ Atoms } & \multirow{2}{*}{$\begin{array}{c}\text { Angle } /^{\circ} \\
89.5(3)\end{array}$} & \multicolumn{3}{|c|}{ Atoms } & \multirow{2}{*}{$\begin{array}{c}\text { Angle }^{\circ} \\
91.6(13)\end{array}$} \\
\hline C3 & $\mathrm{S} 1$ & $\mathrm{C} 2 \mathrm{~A}$ & & $\mathrm{~F} 2 \mathrm{~A}$ & $\mathrm{C} 11$ & F3A & \\
\hline $\mathrm{C} 3$ & $\mathrm{~S} 1$ & $\mathrm{C} 2 \mathrm{~B}$ & $90.7(6)$ & $\mathrm{F} 2 \mathrm{~A}$ & $\mathrm{C} 11$ & $\mathrm{C} 8$ & $117.5(11)$ \\
\hline $\mathrm{C} 4$ & N1 & $\mathrm{N} 2$ & $117.1(5)$ & F3A & $\mathrm{C} 11$ & $\mathrm{C} 8$ & $112.2(7)$ \\
\hline N1 & N2 & $\mathrm{C} 1$ & $127.0(4)$ & F1B & C11 & F3B & $131(2)$ \\
\hline $\mathrm{C} 3$ & $\mathrm{~N} 2$ & N1 & $116.4(4)$ & F1B & $\mathrm{C} 11$ & $\mathrm{C} 8$ & $116(2)$ \\
\hline $\mathrm{C} 3$ & N2 & $\mathrm{C} 1$ & $116.5(4)$ & F2B & $\mathrm{C} 11$ & F1B & $87.5(19)$ \\
\hline $\mathrm{N} 2$ & $\mathrm{C} 1$ & $\mathrm{C} 2 \mathrm{~A}$ & $105.1(5)$ & $\mathrm{F} 2 \mathrm{~B}$ & $\mathrm{C} 11$ & F3B & $87.3(17)$ \\
\hline $\mathrm{N} 2$ & $\mathrm{C} 1$ & $\mathrm{C} 2 \mathrm{~B}$ & $106.3(8)$ & $\mathrm{F} 2 \mathrm{~B}$ & $\mathrm{C} 11$ & $\mathrm{C} 8$ & $116.8(15)$ \\
\hline $\mathrm{N} 2$ & $\mathrm{C} 3$ & $\mathrm{~S} 1$ & 113.3(4) & F3B & $\mathrm{C} 11$ & $\mathrm{C} 8$ & $110.2(9)$ \\
\hline $\mathrm{N} 3$ & $\mathrm{C} 3$ & $\mathrm{~S} 1$ & $123.1(4)$ & $\mathrm{C} 13$ & $\mathrm{C} 12$ & $\mathrm{C} 17$ & $118.6(6)$ \\
\hline N3 & $\mathrm{C} 3$ & $\mathrm{~N} 2$ & $123.6(5)$ & $\mathrm{C} 13$ & $\mathrm{C} 12$ & $\mathrm{C} 2 \mathrm{~A}$ & $116.6(5)$ \\
\hline N1 & $\mathrm{C} 4$ & C5 & 119.1(5) & $\mathrm{C} 13$ & $\mathrm{C} 12$ & $\mathrm{C} 2 \mathrm{~B}$ & 118.9(9) \\
\hline C6 & $\mathrm{C} 5$ & $\mathrm{C} 4$ & $119.9(5)$ & $\mathrm{C} 17$ & $\mathrm{C} 12$ & $\mathrm{C} 2 \mathrm{~A}$ & $124.0(6)$ \\
\hline C6 & $\mathrm{C} 5$ & $\mathrm{C} 10$ & $118.8(5)$ & $\mathrm{C} 17$ & $\mathrm{C} 12$ & $\mathrm{C} 2 \mathrm{~B}$ & $111.8(9)$ \\
\hline $\mathrm{C} 10$ & $\mathrm{C} 5$ & $\mathrm{C} 4$ & $121.3(5)$ & $\mathrm{C} 14$ & $\mathrm{C} 13$ & $\mathrm{C} 12$ & $120.8(6)$ \\
\hline $\mathrm{C} 7$ & C6 & $\mathrm{C} 5$ & $121.2(5)$ & $\mathrm{C} 13$ & $\mathrm{C} 14$ & $\mathrm{C} 15$ & $119.6(6)$ \\
\hline C6 & $\mathrm{C} 7$ & $\mathrm{C} 8$ & $119.7(5)$ & $\mathrm{C} 16$ & $\mathrm{C} 15$ & $\mathrm{C} 14$ & $119.8(6)$ \\
\hline $\mathrm{C} 7$ & $\mathrm{C} 8$ & $\mathrm{C} 11$ & $120.6(6)$ & $\mathrm{C} 17$ & $\mathrm{C} 16$ & $\mathrm{C} 15$ & $120.7(6)$ \\
\hline C9 & $\mathrm{C} 8$ & $\mathrm{C} 7$ & $119.8(5)$ & $\mathrm{C} 16$ & $\mathrm{C} 17$ & $\mathrm{C} 12$ & $120.5(6)$ \\
\hline C9 & $\mathrm{C} 8$ & $\mathrm{C} 11$ & $119.6(6)$ & $\mathrm{C} 1$ & $\mathrm{C} 2 \mathrm{~A}$ & $\mathrm{~S} 1$ & $106.9(5)$ \\
\hline $\mathrm{C} 10$ & C9 & $\mathrm{C} 8$ & $120.4(5)$ & $\mathrm{C} 1$ & $\mathrm{C} 2 \mathrm{~A}$ & $\mathrm{C} 12$ & $113.0(6)$ \\
\hline C9 & $\mathrm{C} 10$ & C5 & $120.1(5)$ & $\mathrm{C} 12$ & $\mathrm{C} 2 \mathrm{~A}$ & S1 & $111.4(5)$ \\
\hline F1A & C11 & F3A & $99.4(7)$ & $\mathrm{C} 1$ & $\mathrm{C} 2 \mathrm{~B}$ & $\mathrm{~S} 1$ & $101.0(10)$ \\
\hline F1A & C11 & $\mathrm{C} 8$ & $114.7(6)$ & $\mathrm{C} 12$ & $\mathrm{C} 2 \mathrm{~B}$ & $\mathrm{~S} 1$ & $109.9(11)$ \\
\hline F2A & C11 & F1A & $116.9(13)$ & $\mathrm{C} 12$ & $\mathrm{C} 2 \mathrm{~B}$ & $\mathrm{C} 1$ & $110.8(12)$ \\
\hline
\end{tabular}


J. Chil. Chem. Soc., 64, N² (2019)

Table 4. Bond Lengths for the title compound

\begin{tabular}{|c|c|c|c|c|c|}
\hline \multicolumn{2}{|c|}{ Atoms } & \multirow{2}{*}{$\begin{array}{c}\text { Length/§ } \\
1.729(5) \\
\end{array}$} & \multicolumn{2}{|c|}{ Atoms } & \multirow{2}{*}{$\begin{array}{c}\text { Length/Å } \\
1.474(7) \\
\end{array}$} \\
\hline $\mathrm{S} 1$ & $\mathrm{C} 3$ & & $\mathrm{C} 4$ & $\mathrm{C} 5$ & \\
\hline $\mathrm{S} 1$ & $\mathrm{C} 2 \mathrm{~A}$ & $1.826(8)$ & $\mathrm{C} 5$ & C6 & $1.386(7)$ \\
\hline $\mathrm{S} 1$ & $\mathrm{C} 2 \mathrm{~B}$ & $1.89(2)$ & $\mathrm{C} 5$ & $\mathrm{C} 10$ & $1.401(8)$ \\
\hline F1A & $\mathrm{C} 11$ & $1.334(8)$ & C6 & $\mathrm{C} 7$ & $1.375(8)$ \\
\hline F2A & $\mathrm{C} 11$ & $1.316(12)$ & $\mathrm{C} 7$ & $\mathrm{C} 8$ & $1.389(8)$ \\
\hline F3A & $\mathrm{C} 11$ & $1.362(9)$ & $\mathrm{C} 8$ & $\mathrm{C} 9$ & $1.388(8)$ \\
\hline F1B & $\mathrm{C} 11$ & $1.356(16)$ & $\mathrm{C} 8$ & $\mathrm{C} 11$ & $1.458(7)$ \\
\hline $\mathrm{F} 2 \mathrm{~B}$ & $\mathrm{C} 11$ & $1.347(14)$ & $\mathrm{C} 9$ & $\mathrm{C} 10$ & $1.376(8)$ \\
\hline F3B & $\mathrm{C} 11$ & $1.366(12)$ & $\mathrm{C} 12$ & $\mathrm{C} 13$ & $1.387(8)$ \\
\hline O1S & C1S & $1.344(11)$ & $\mathrm{C} 12$ & $\mathrm{C} 17$ & $1.391(8)$ \\
\hline N1 & $\mathrm{N} 2$ & $1.378(6)$ & $\mathrm{C} 12$ & $\mathrm{C} 2 \mathrm{~A}$ & $1.560(9)$ \\
\hline N1 & $\mathrm{C} 4$ & $1.266(7)$ & $\mathrm{C} 12$ & $\mathrm{C} 2 \mathrm{~B}$ & $1.53(2)$ \\
\hline $\mathrm{N} 2$ & $\mathrm{C} 1$ & $1.456(7)$ & $\mathrm{C} 13$ & $\mathrm{C} 14$ & $1.374(8)$ \\
\hline $\mathrm{N} 2$ & $\mathrm{C} 3$ & $1.335(7)$ & $\mathrm{C} 14$ & $\mathrm{C} 15$ & $1.381(9)$ \\
\hline N3 & $\mathrm{C} 3$ & $1.296(7)$ & $\mathrm{C} 15$ & $\mathrm{C} 16$ & $1.377(10)$ \\
\hline $\mathrm{C} 1$ & $\mathrm{C} 2 \mathrm{~A}$ & $1.508(9)$ & $\mathrm{C} 16$ & $\mathrm{C} 17$ & $1.359(8)$ \\
\hline $\mathrm{C} 1$ & $\mathrm{C} 2 \mathrm{~B}$ & $1.581(19)$ & & & \\
\hline
\end{tabular}

In the crystal $\mathrm{N}-\mathrm{H} \cdots \mathrm{Br}$ hydrogen bonds link the components into a bi-dimensional network with the cations and anions stacked parallel to plane 101 . The weak interactions are mainly constituted by $\mathrm{H} \cdots \mathrm{F}, \mathrm{H} \cdots \pi$ and $\mathrm{H} \cdots \mathrm{Br}$. Moreover, In crystal structure it is found strong charge assisted hydrogen-halogen bonding and, intermolecular hydrogen- $\pi$ bonding interaction along to [010] direction with $2.40 \AA$ (Figure 2 and table 5)
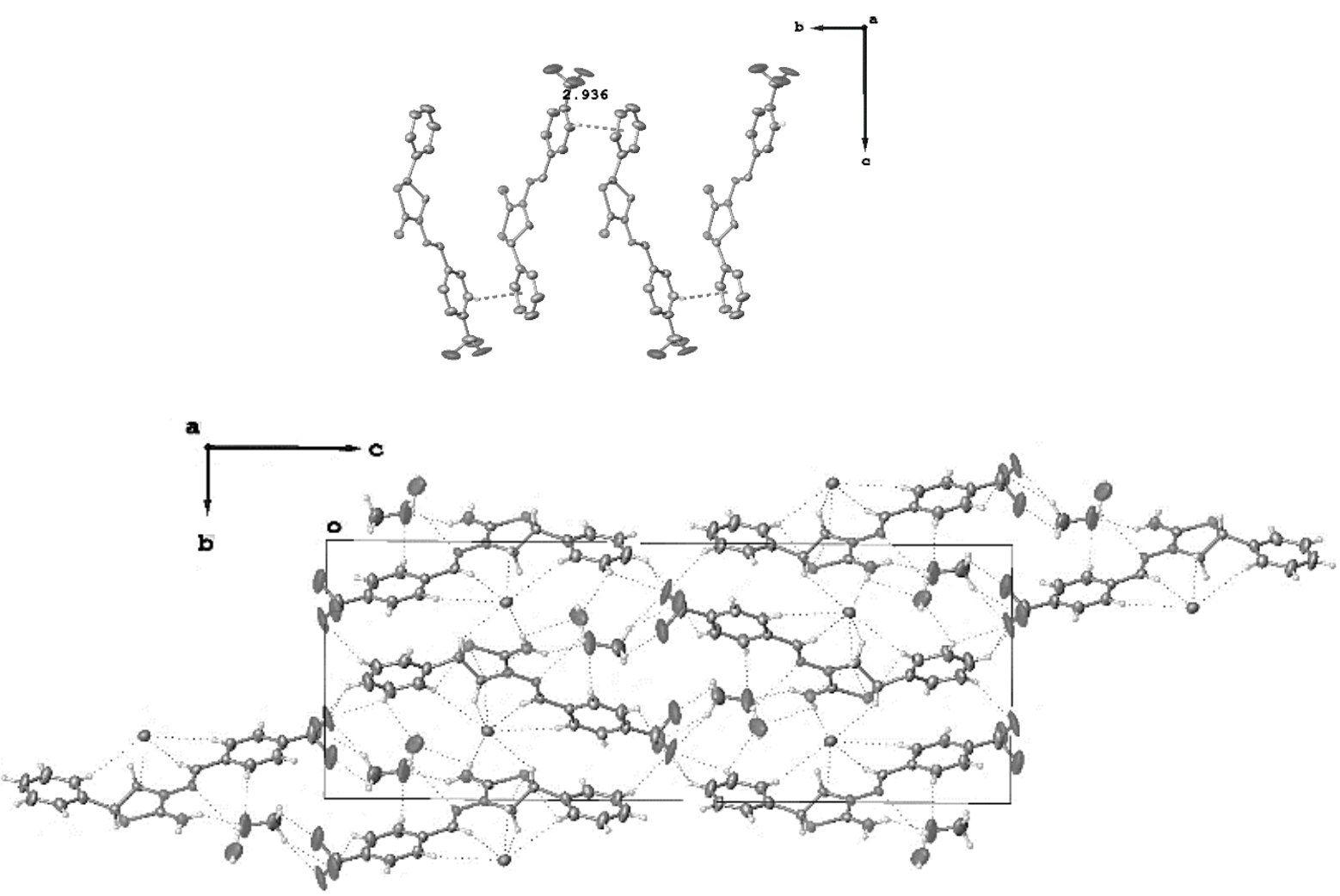

Figure 2. Non-covalent interactions in the crystal structure of the title compound. 
Table 5. Hydrogen Bonds for Gul7.

\begin{tabular}{|c|c|c|c|c|c|}
\hline $\mathbf{D}$ & $\mathbf{H}$ & $\mathbf{A}$ & $\mathbf{d}(\mathbf{D}-\mathbf{H}) / \AA$ & $\mathbf{d}(\mathbf{H}-\mathbf{A}) / \AA$ & $\mathbf{D}-\mathbf{H}-\mathbf{A} /{ }^{\circ}$ \\
\hline $\mathrm{O} 1 \mathrm{~S}$ & $\mathrm{H} 1 \mathrm{~S}$ & $\mathrm{O} 2 \mathrm{~S}^{1}$ & 0.84 & 2.21 & $\mathbf{d}(\mathbf{D}-\mathbf{A}) / \mathbf{\AA}$ \\
\hline $\mathrm{N} 3$ & $\mathrm{H} 3 \mathrm{~A}$ & $\mathrm{O} 1 \mathrm{~S}$ & 0.88 & 2.10 & $2.991(16)$ \\
\hline
\end{tabular}

$1-1+\mathrm{X},+\mathrm{Y},+\mathrm{Z}$

A Hirshfeld surface analysis was conducted to verify the contributions of the different intermolecular interactions. This analysis was used to investigate the presence of hydrogen bonds and intermolecular interactions in the crystal structure. The Hirshfeld surface analysis ${ }^{26}$ was generated by CrystalExplorer $17.5^{27}$ and comprised $\mathrm{d}_{\text {norm }}$ surface plots and $2 \mathrm{D}$ (two-dimensional) fingerprint plots ${ }^{28}$. The plots of the Hirshfeld surface confirms the presence of the non-covalent interaction described below (Figure 3.), taking account the several positional disorder in the molecular structure
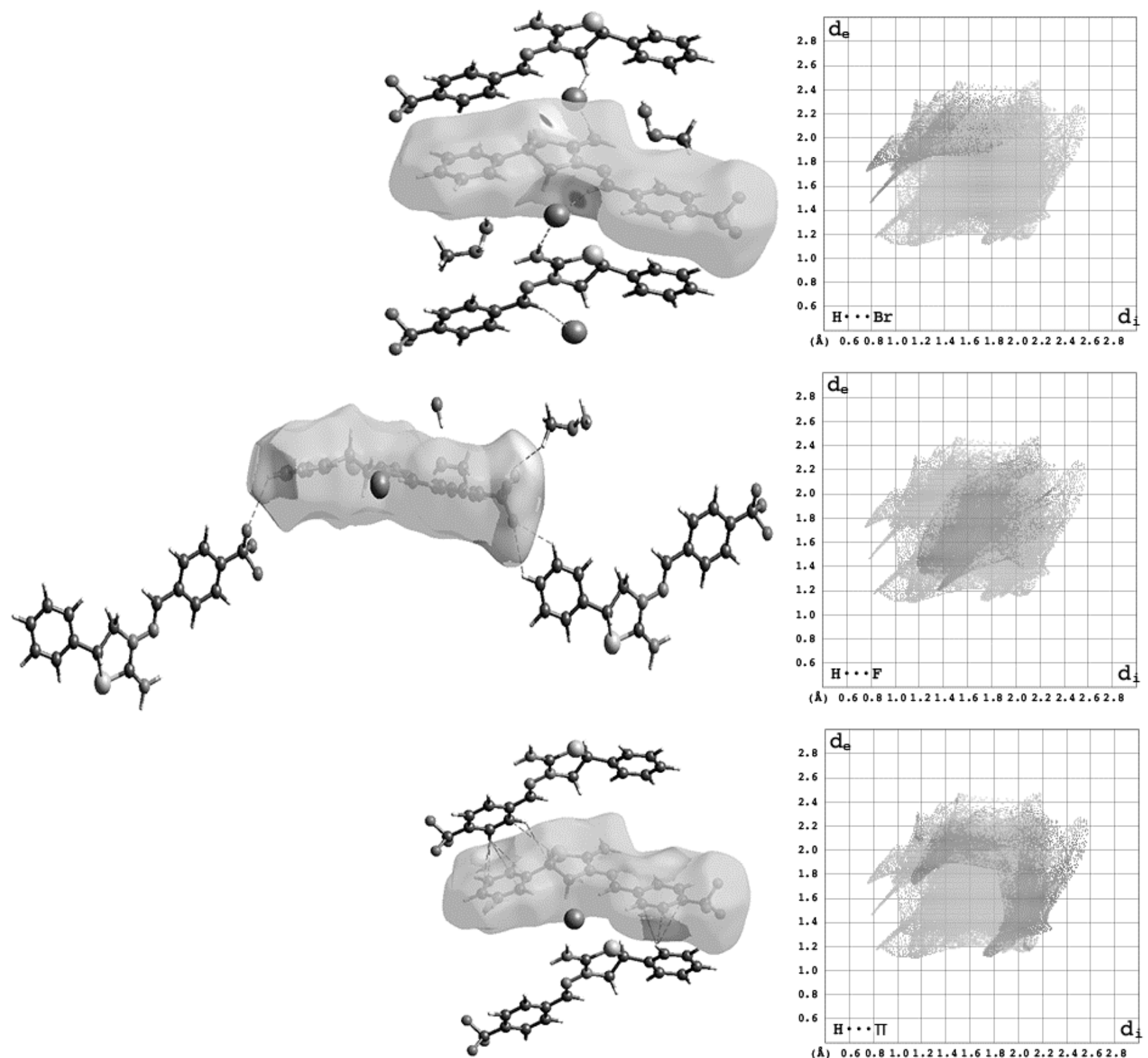

Figure 3. The two-dimensional fingerprint plots of the title compound $\left[d_{e}\right.$ and $d_{i}$ represent the distances from a point on the Hirshfeld surface to the nearest atoms outside (external) and inside (internal) the surface, respectively]. 
Table 6. Contributions of interatomic contacts to the Hirshfeld surface for the title compound*.

\begin{tabular}{|l|l|}
\hline Contact & Contribution (\%) \\
\hline $\mathrm{Br} \cdots \mathrm{H}$ & $6.8 \%$ \\
\hline $\mathrm{Br} \cdots \mathrm{N}$ & $0.9 \%$ \\
\hline $\mathrm{Br} \cdots \mathrm{C}$ & $0.7 \%$ \\
\hline $\mathrm{F} \cdots \mathrm{F}$ & $0.5 \%$ \\
\hline $\mathrm{F} \cdots \mathrm{O}$ & $0.1 \%$ \\
\hline $\mathrm{F} \cdots \mathrm{H}$ & $24.5 \%$ \\
\hline $\mathrm{F} \cdots \mathrm{C}$ & $1.0 \%$ \\
\hline $\mathrm{O} \cdots \mathrm{H}$ & $2.9 \%$ \\
\hline $\mathrm{O} \cdots \mathrm{C}$ & $0.3 \%$ \\
\hline $\mathrm{H} \cdots \mathrm{H}$ & $32.8 \%$ \\
\hline $\mathrm{H} \cdots \mathrm{N}$ & $3.8 \%$ \\
\hline $\mathrm{H} \cdots \mathrm{C}(\pi-\mathrm{H})$ & $19.3 \%$ \\
\hline
\end{tabular}

*Reciprocal contacts

The weak intermolecular interactions are mainly constituted by $\mathrm{H} \cdots \mathrm{F}, \mathrm{H} \cdots \pi$ and $\mathrm{H} \cdots \mathrm{Br}$, with a contribution of 24.5 , 19.3 and $6.8 \%$, respectively. Where the reciprocal contacts appear as a wide stain for $\mathrm{H} \cdots \mathrm{F}$, with $d_{\mathrm{e}}+d_{\mathrm{i}} \simeq 2.8 \AA$, for $\mathrm{H} \cdots \mathrm{Br}$ as a sharp spike with $d_{\mathrm{e}}+d_{\mathrm{i}} \simeq 2.3 \AA$ and, $\pi \cdots \mathrm{H}$ as asymmetrical wings with $d_{\mathrm{e}}+$ $d_{\mathrm{i}} \simeq 3.0$. A, according to the interaction depicted in figure 2 . This type of weak interactions are also observed in isostructural compounds recently reported ${ }^{23-25}$.

\section{CONCLUSIONS.}

In this study we offer the report of synthesis, characterization and structural studies of the title compounds, showing the $E$ isomer in the solid state. The weaks intermolecular interactions, were successfully verified with Hirshfeld surface analyses, being mainly constituted by $\mathrm{H} \cdots \mathrm{F}, \mathrm{H} \cdots \pi$ and $\mathrm{H} \cdots \mathrm{Br}$, with a contribution of $24.5,19.3$ and $6.8 \%$, respectively. The chemistry of this compound allow postulate as a good candidate for several applications such as potential biological, pharmacological and analytical applications, moreover heterocyclic amines are also widely used in the synthesis of Schiff bases, which provide different kinds of noncovalent interactions.

\section{ACKNOWLEDGEMENTS.}

Ali Khalilov thankful to Baku State University for the "50+50" individual grant support in this work. Iván Brito, Alejandro Cárdenas and Jonathan Cisterna, thank to Universidad de Antofagasta for purchase license for the Cambridge Structural Database and for the financial support. Jonathan Cisterna acknowledge to Universidad de Antofagasta for postdoctoral fellowship.

\section{REFERENCES.}

1. G. Mahmoudi, F. A. Afkhami, A. Castiñeiras, I. García-Santos, A. Gurbanov, F. I. Zubkov, M. P. Mitoraj, M. Kukułka, F. Sagan, D. W. Szczepanik, I. A. Konyaeva and D. A. Safin, Inorg. Chem., 2018, 57, 43954408 .

2. G. Mahmoudi, A. Bauzá, A. V. Gurbanov, F. I. Zubkov, W. Maniukiewicz, A. Rodríguez-Diéguez, E. López-Torres and A. Frontera, CrystEngComm, 2016, 18,9056-9066.

3. K. T. Makhmudov, R. A. Alieva, S. R. Gadzhieva and F. M. Chyragov, J. Anal. Chem., 2008, 63, 435-438.

4. A. M. Maharramov, R. A. Alieva, K. T. Mahmudov, A. V Kurbanov and R. K. Askerov, Russ. J. Coord. Chem., 2009, 35, 704-709.

5. D. Y. Vandyshev, K. S. Shikhaliev, A. Y. Potapov, M. Y. Krysin, F. I. Zubkov and L. V. Sapronova, Beilstein J. Org. Chem., 2017, 13, 2561-2568.

6. D. K. Nasirova, A. V. Malkova, K. B. Polyanskii, K. Y. Yankina, P. N. A. Amoyaw, I. A. Kolesnik, A. V. Kletskov, I. A. Godovikov, E. V. Nikitina and F. I. Zubkov, Tetrahedron Lett., 2017, 58, 4384-4387.

7. E. A. Kvyatkovskaya, V. P. Zaytsev, F. I. Zubkov, P. V. Dorovatovskii, Y. V. Zubavichus and V. N. Khrustalev, Acta Crystallogr. Sect. E Crystallogr. Commun., 2017, 73, 515-519.

8. A. A. Shetnev and F. I. Zubkov, Chem. Heterocycl. Compd., 2017, 53, 495497.

9. A. V. Gurbanov, A. M. Maharramov, F. I. Zubkov, A. M. Saifutdinov and F. I. Guseinov, Aust. J. Chem., 2018, 71, 190-194.
10. K. T. Mahmudov, M. N. Kopylovich, A. Sabbatini, M. G. B. Drew, M. D. R. S. Martins, C. Pettinari and A. J. L. Pombeiro, Inorg. Chem., 2014, 53, 9946-9958.

11. R. Jlassi, A. P. C. Ribeiro, M. F. C. Guedes Da Silva, K. T. Mahmudov, M. N. Kopylovich, T. B. Anisimova, H. Naïli, G. A. O. Tiago and A. J. L. Pombeiro, Eur. J. Inorg. Chem., 2014, 2014, 4541-4550.

12. A. V. Gurbanov, G. Mahmoudi, M. F. C. Guedes da Silva, F. I. Zubkov, K. T. Mahmudov and A. J. L. Pombeiro, Inorganica Chim. Acta, 2018, 471, $130-136$.

13. N. Q. Shikhaliyev, N. E. Ahmadova, A. V. Gurbanov, A. M. Maharramov, G. Z. Mammadova, V. G. Nenajdenko, F. I. Zubkov, K. T. Mahmudov and A. J. L. Pombeiro, Dye. Pigment., 2018, 150, 377-381.

14. A. V. Gurbanov, K. T. Mahmudov, M. N. Kopylovich, F. M. Guedes da Silva, M. Sutradhar, F. I. Guseinov, F. I. Zubkov, A. M. Maharramov and A. J. L. Pombeiro, Dye. Pigment., 2017, 138, 107-111.

15. K. T. Mahmudov and A. J. L. Pombeiro, Chem. - A Eur. J., 2016, 22, 1635616398.

16. K. T. Mahmudov, M. N. Kopylovich, M. F. C. Guedes Da Silva and A. J. L. Pombeiro, Dalt. Trans., 2017, 46, 10121-10138.

17. J. D. Van der Waals, University of Leiden, Leiden., 1873.

18. Bruker AXS INC., APEX3, SAINT and SADABS, 2016, Bruker AXS Inc., Madison, Wisconsin, USA.

19. G. M. Sheldrick, Acta Crystallogr. Sect. C Struct. Chem., 2015, 71, 3-8.

20. O. V Dolomanov, L. J. Bourhis, R. J. Gildea, J. A. K. Howard and H. Puschmann, J. Appl. Crystallogr., 2009, 42, 339-341.

21. C. D. Centre and L. Road, 1986, 13, 4343-4348.

22. F. H. Allen, O. Kennard, D. G. Watson, L. Brammer and A. G. Orpen, 1987, $1-19$.

23. A.N.Khalilov, Z.Atioğlu, M.Akkurt, G.S.Duruskari, F.A.A.Toze and A.T.Huseynova. Acta Cryst., 2019, E75, 662-666

24. M.Akkurt, G.Sh.Duruskari, F.A.A.Toze, A.N.Khalilov and A.T.Huseynova. Acta Cryst., 2018, E74, 1168-1172.

25. M.Akkurt, A.M.Maharramov, G.S.Duruskari, F.A.A.Toze and A.N.Khalilov. Acta Cryst., 2018, E74, 1290-1294.

26. J. J. McKinnon, D. Jayatilaka and M. A. Spackman, Chem. Commun., 2007, 3814-3816.

27. M. A. Wolff, S.K., Grimwood, D.J., McKinnon, J.J., Turner, M.J., Jayatilaka, D. and Spackman, 2012, Crystal Explorer 17.5. University of Western Austr.

28. M. A. Spackman and J. J. McKinnon, CrystEngComm, 2002, 4, 378-392. 